\title{
Alterstice
}

Revue internationale de la recherche interculturelle

International Journal of Intercultural Research

Revista International de la Investigacion Intercultural

\section{Retombées et perspectives de la " diversité " et des politiques linguistiques en Vallée d'Aoste : le cas du français et du francoprovençal}

\section{Maria Immacolata Spagna}

Volume 7, numéro 2, 2017

Diversité(s) au coeur des politiques et des pratiques : entre l'Europe et l'Afrique

URI : https://id.erudit.org/iderudit/1052566ar

DOI : https://doi.org/10.7202/1052566ar

Aller au sommaire du numéro

Éditeur(s)

Alterstice

ISSN

1923-919X (numérique)

Découvrir la revue

Citer cet article

Spagna, M. I. (2017). Retombées et perspectives de la « diversité » et des politiques linguistiques en Vallée d'Aoste : le cas du français et du francoprovençal. Alterstice, 7(2), 11-21. https://doi.org/10.7202/1052566ar

\section{Résumé de l'article}

Cette contribution se propose de mettre en lumière les retombées des politiques linguistiques mises en oeuvre au cours des siècles en Vallée d'Aoste sur la communauté valdôtaine. Suivant les changements du statut du français et du francoprovençal, causés par les mesures imposées par l'État depuis l'Unité italienne, nous relèverons l'évolution de la perception des Valdôtains face à ces choix politiques. Si, jusqu'en 1861, les Valdôtains se considéraient de nationalité française, à l'intérieur du nouvel État italien, notamment à la suite de la dictature de Mussolini contre le français, ils se retrouvèrent sans aucune garantie juridique de leur identité linguistique. En fait, le Statut Spécial n’a presque rien changé. Les récentes enquêtes sociolinguistiques attestent que l'intérêt pour la survie de la diversité linguistique est désormais très faible. Dans une telle situation, le plurilinguisme, vers lequel s'orientent les politiques actuelles, pourra produire les résultats souhaités seulement s'il n'est pas imposé mais plutôt favorisé, dès l'enfance, par le biais d'approches plurielles. 


\section{7}

alterstice

Revue Internationale

de la Recherche Interculturelle

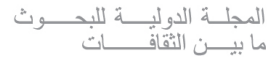

Revista International

de la Investigacion Intercultural

Revista Internacional de Pesquisa Intercultural
International Journal of Intercultural Research

ARTICLE THÉMATIQUE

\section{Retombées et perspectives de la " diversité » et des politiques linguistiques en Vallée d'Aoste : le cas du français et du francoprovençal}

Maria Immacolata Spagna ${ }^{1}$

\section{Résumé}

Cette contribution se propose de mettre en lumière les retombées des politiques linguistiques mises en œuvre au cours des siècles en Vallée d'Aoste sur la communauté valdôtaine. Suivant les changements du statut du français et du francoprovençal, causés par les mesures imposées par l'État depuis l'Unité italienne, nous relèverons l'évolution de la perception des Valdôtains face à ces choix politiques. Si, jusqu'en 1861, les Valdôtains se considéraient de nationalité française, à l'intérieur du nouvel État italien, notamment à la suite de la dictature de Mussolini contre le français, ils se retrouvèrent sans aucune garantie juridique de leur identité linguistique. En fait, le Statut Spécial n'a presque rien changé. Les récentes enquêtes sociolinguistiques attestent que l'intérêt pour la survie de la diversité linguistique est désormais très faible. Dans une telle situation, le plurilinguisme, vers lequel s'orientent les politiques actuelles, pourra produire les résultats souhaités seulement s'il n'est pas imposé mais plutôt favorisé, dès l'enfance, par le biais d'approches plurielles.

\section{Rattachement de l'auteure \\ ${ }^{1}$ Université du Salento, Italie}

\section{Correspondance}

mariaimmacolata.spagna@unisalento.it

\section{Mots clés}

politiques linguistiques, français, francoprovençal, diversité linguistique, plurilinguisme

\section{Pour citer cet article}

Spagna, M. I. (2017). Retombées et perspectives de la "diversité " et des politiques linguistiques en Vallée d'Aoste : le cas du français et du francoprovençal. Alterstice, 7(2), 11-22. 


\section{Introduction}

Région officiellement bilingue italien-français, la Vallée d'Aoste se caractérise par un plurilinguisme diffus où les deux langues officielles cohabitent avec le francoprovençal, le walser et des dialectes italiens comme le piémontais, le calabrais, le vénitien, etc. Ce plurilinguisme, confirmé par le sondage linguistique de la Fondation Émile Chanoux $(2001)^{1}$, fait de la Vallée d'Aoste un lieu de rencontre interculturelle, de contact mais aussi de tensions et de conflits, avec une réalité caractérisée par une dynamique entre groupes différents, marquée par des rapports de force entre les revendications identitaires. Comme le rappelle Calvet (1987, p. 153, 282), « derrière les rapports de langues, ce sont des rapports sociaux que l'on trouve" et "les conflits linguistiques nous parlent de conflits sociaux ", car les langues ne sont pas seulement un moyen de communication, mais aussi et surtout les vecteurs d'une identité et donc les vecteurs clés de la diversité.

La position géographique de la Vallée d'Aoste, au nord-ouest de l'Italie, qui en a fait un état intramontain et de carrefour, a eu une influence considérable sur sa situation linguistique : elle a favorisé des voies de passage aux langues de l'aire francophone et, par conséquent, la formation de sa propre personnalité et de son particularisme.

Cette contribution, conçue dans une approche transdisciplinaire - sociolinguistique et analyse du discours - se propose de mettre en lumière comment la situation actuelle du français et du francoprovençal en Vallée d'Aoste n'est en fait que le résultat des tensions issues de l'histoire entre politiques linguistiques et comportements sociolinguistiques et identitaires individuels des Valdôtains.

Voilà pourquoi nous présenterons les mesures de politique linguistique les plus importantes adoptées en Vallée d'Aoste, depuis l'édit de Rivoli jusqu'à la loi 482/1999, afin de suivre l'évolution de la perception des Valdôtains face à ces choix politiques imposés à leur langue et, donc, à leur identité collective. Ce parcours nous permettra ainsi de mieux comprendre la situation actuelle du français et du francoprovençal, attestée par les récentes enquêtes sociolinguistiques sur lesquelles nous allons nous appuyer, pour entrevoir ensuite les perspectives pour ces deux langues.

\section{Les Valdôtains, leur langue et les politiques linguistiques en Vallée d’Aoste}

\section{La maison de Savoie et l'édit de Rivoli}

Comme le remarque Zanotto (1986, p. 14-15), la francisation de la Vallée commence à partir de 575, date où les Lombards cédèrent la Vallée d'Aoste au roi Gontran, qui la réunit au royaume des Francs : depuis lors, elle reçut une orientation précise quant aux caractéristiques ethniques et linguistiques. En particulier, le parler valdôtain allait subir de profondes transformations sous l'influence de l'Ouest: le parler local allait devenir le francoprovençal, c'est-à-dire un parler appartenant au domaine français qui, n'ayant jamais atteint l'état de koïné, s'est décliné en une variété de dialectes. Quand, dans les premières décennies du $11^{\mathrm{e}}$ siècle, fit son apparition sur la scène valdôtaine la maison de Savoie, il était tout à fait naturel que le français devienne la langue écrite, tout comme dans le Lyonnais, en Savoie et en Suisse romande.

Le premier acte de politique linguistique remonte à 1561, date du célèbre édit du Duc de Savoie Emmanuel Philibert, qui imposa le français comme seule langue des actes officiels du Pays d'Aoste, à la place du latin. Cette décision fut prise dans le but de faciliter la compréhension de ces actes par le peuple, en raison du fait que la plupart des Valdôtains étaient francophones et que le français était une langue « plus commune, et générale que point d'autre ", puisque les Valdôtains étaient un peuple " acoutumé de parler la dite langue plus aisément que nulle autre » (cité dans Puolato, 2006, p. 10).

\footnotetext{
${ }^{1}$ II s'agit d'une recherche quantitative réalisée sur un échantillon représentatif de la population valdôtaine (7 500 personnes interviewées) au moyen d'un questionnaire à réponses fermées (des oui/non, des croix), exception faite pour la question "Quelle est votre langue maternelle?». Les données du sondage linguistique ont été commentées au cours du colloque scientifique Une Vallée d'Aoste bilingue dans une Europe plurilingue / Una Valle d'Aosta bilingue in un'Europa plurilingue qui s'est déroulé à Aoste du 21 au 23 novembre 2002 et dont les actes ont été publiés en 2003 (lannaccaro, Dell'Aquila et Andrione, 2003).
} 
Au cours des siècles suivants, le français se répandit par les écoles. Même les gens des classes modestes ou des milieux paysans accédèrent à l'enseignement de cette langue grâce aux écoles de hameau mais, malgré une connaissance du français, ils parlaient le patois: "Le français était la langue de la noblesse [...]. Les classes modestes du Val d'Aoste devaient parler le patois (franco-provençal) " (Janin, 1991, p. 135). Le francoprovençal et le français ont, donc, vécu côte à côte sur le mode d'une diglossie. Le français, expression d'une littérature appréciée, qui possédait déjà une écriture, permettait la communication avec les autres régions du Duché de Savoie et était, notamment, langue officielle des écrits et des actes solennels, publics et religieux, jouissant du prestige de la variété noble, alors que le patois, utilisé pour la conversation spontanée et informelle, représentait la variété basse (Cavalli, 1997). Cette situation linguistique est décrite par la formule suivante: "le patois est la langue de tous les jours et le français, la langue du dimanche ", remarquant le fait que le sermon dominical était prononcé en français (Tuaillon, 1992, p. 15).

L'option sans condition en faveur du français a fait en sorte qu'on n'attribue jamais au patois le rôle de représentant de la culture et de la personnalité valdôtaine (Omezzoli, 1995), même si sa pénétration dans les différentes classes sociales était favorisée par le fait qu'il n'y avait pas, à son égard, une interdiction sociale ou esthétique. En effet, comme le souligne Bétemps $(1978$, p. 8), «En Vallée d’Aoste personne n'avait jamais perçu une opposition quelconque entre le français et le patois. Le français n'était autre que la langue de grande communication dont le patois n'était qu'un dialecte ". La seule menace était représentée par le piémontais, vu le transfert en 1562 du centre administratif du Duché de Savoie de Chambéry à Turin. C'est par l'administration de Turin que l'italien allait s'infiltrer (Schüle, 1990).

Cette situation linguistique, où la langue-toit française - au sens "klossien » du terme - était bien solide et prédominante et la position de l'italien encore assez faible (Bauer, 1999, p. 79), dura jusqu'à ce que la Vallée fit partie du Duché de Savoie. La population était presque totalement francophone (plus de $93 \%$, selon le recensement linguistique de 1861). En effet, "Avant 1861, les Valdôtains semblent se considérer de nationalité française, tout court ... et parfois de nationalité valdôtaine » (Bétemps, 1979, p. 51-52).

\section{L'Unité d'Italie et le début du déclin du français}

Après l'annexion de la Savoie à la France et la proclamation de l'Unité italienne, le nombre des sujets francophones de l'État était réduit aux Valdôtains, soit moins de 100000 personnes, et le gouvernement n'avait aucun intérêt à prendre en considération la question linguistique d'une si petite minorité. Suivant l'idéal de l'État-Nation qui tendait à associer un même territoire, une seule organisation politico-administrative et une langue unique, les nationalistes considéraient comme une anomalie le fait d'avoir dans le territoire national une autre langue dont il fallait tenir compte. En 1861, dans son pamphlet nationaliste très agressif, Diritto e necessità di abrogare il francese come lingua ufficiale in alcune valli della provincia di Torino, le député piémontais Vegezzi Ruscalla soutenait que la Vallée d'Aoste n'était pas française du point de vue linguistique puisque c'était le patois qu'on y parlait (Bétemps, 1978). De plus, il définit la langue française comme une "souillure à effacer " (cité dans lannaccaro, Dell'Aquila et Andrione, 2003, p. 7). Depuis ce moment, afin d'aligner sur le plan linguistique la Vallée au reste du pays, les interventions visant à abolir le français furent nombreuses. L'enseignement de cette langue fut brusquement supprimé au collège Saint-Bénin et, immédiatement, tous les Valdôtains s'unirent pour protester contre cette mesure. Peu à peu l'italien gagnait du terrain non seulement dans l'enseignement, mais aussi dans la vie quotidienne. Si le français, en tant que langue de prestige, était utilisé par la noblesse, par l'Église et par les gens de culture, et si le peuple se contentait de comprendre le français et de parler le francoprovençal ${ }^{2}$, l'italien s'imposa dans toutes les classes sociales.

\footnotetext{
2 À partir de l'an 1873, grâce à la déclaration de G.I. Ascoli, en tête des Schizzi franco-provenzali (1878, p. 61), le francoprovençal est considéré comme un groupe linguistique autonome par rapport au français.
} 
L'unité italienne représenta ainsi pour la Vallée d'Aoste un véritable bouleversement linguistique, qui marqua le début du progressif processus d'italianisation et de déclin du français, jusque là langue officielle dans la région. Comme le souligne Bétemps (1974, p. 12) :

le français fut « imposé » par E. Philibert en opposition au latin, une langue morte depuis longtemps, l'italien fut imposé en opposition au français, langue toit du francoprovençal, une langue bien vivante en Vallée d’Aoste profondément enracinée dans le tissu social valdôtain.

Voilà l'origine de la question valdôtaine, qui trouva dans la langue française l'élément constitutif d'une diversité depuis lors toujours confirmée. Le français devenait le nœud du particularisme valdôtain, le symbole de l’identité valdôtaine.

C'était dans le but de conserver la langue française qu'en 1909 naissait la Ligue valdôtaine, première expression du sentiment autonomiste, fondée par Anselme Réan. À côté de la lutte pour la conservation de la langue française, les Valdôtains devenaient de plus en plus conscients qu'ils représentaient une entité particulière dans le cadre de l'État italien, et leurs aspirations autonomistes commençaient à prendre forme.

\section{Le fascisme de Mussolini et la destruction du français}

En $1921^{3}$, $88 \%$ de la population était encore francophone. Le processus d'italianisation mis en place par le nouvel État italien atteignit son aboutissement le plus éclatant à cause des mesures de politique linguistique contre le français de l'époque fasciste, avec, en particulier, l'interdiction sous peine d'amende de l'emploi de mots étrangers sur les affiches et les enseignes (Décret-loi du 11 février 1923, n³52), l'obligation de rédiger exclusivement en italien les actes de l'état civil (décret 15.10.1925, n 1796), I'abolition de l'enseignement du français dans les écoles (Décret-loi du 22 novembre 1925, $\mathrm{n}^{\circ}$ 2191), l'italianisation de la toponymie (ordonnance du 22.7.1939), l'encouragement d'une immigration massive d'ouvriers venant d'autres régions italiennes, l'incitation implicite à l'émigration de la population autochtone et la suppression de l'usage du français dans la presse (Zanotto, 1986, p. 45).

Le fascisme de Mussolini (de 1922 à 1945) a réalisé ainsi un véritable "nettoyage » aussi bien ethnique que linguistique et culturel. Les appartenants à la minorité étaient désormais sans aucune garantie juridique de leur identité linguistique. En revanche, le francoprovençal, le parler maternel de la majorité de la population, résistait mieux aux assauts. L'attitude du régime vers le patois fut différente puisque, selon Bauer (1999, p. 82), il était moins vulnérable, n'ayant pas de norme écrite. De plus, il était beaucoup plus difficile de contrôler ses domaines d'emploi informels.

Pour réagir aux oppressions mises en œuvre par le régime fasciste, des jeunes gens se réunirent autour de l'abbé Joseph-Marie Trèves et de l'intellectuel Émile Chanoux, donnant naissance à une nouvelle association, La Jeune Vallée d'Aoste (en 1925, le régime avait dissous la Ligue Valdôtaine), afin de soutenir et de défendre les droits, les traditions, la langue et les institutions de la Vallée d’Aoste. Pour les Valdôtains, « la défense de la liberté se confond avec celle du français » (Janin, 1991, p. 494).

Un mouvement sécessionniste prônant l'annexion de la Vallée d'Aoste à la France continua jusqu'à l'approbation du Statut Spécial.

\section{L'après-guerre}

La fin de la Seconde Guerre mondiale marqua un élan d'espoir : on enregistra la volonté politique de réinstaurer ce bilinguisme, existant de façon informelle avant la guerre et éradiqué par Mussolini. Eu égard à sa spécificité, en 1945, l’État italien, notamment grâce à la poussée donnée par la résistance pro-francophone de La Jeune Vallée d'Aoste, concéda à la Vallée un régime d'autonomie de type législatif et économique et l'Assemblée Constituante

\footnotetext{
${ }^{3}$ C'est la date du dernier recensement comportant une question sur la langue, avant le sondage réalisé par la Fondation Chanoux en 2001.
} 
d'Italie adopta le Statut Spécial pour la région, promulgué par la loi constitutionnelle $n^{\circ} 4$ du 26 février 1948 (Zanotto, 1986). En particulier, l'article 38 du Statut reconnut le français comme langue officielle et paritaire, à côté de l'italien; de même, les articles 39 et 40 introduisirent la possibilité d'enseigner le français et certaines disciplines en français dans les écoles.

Le Statut Spécial constituait donc un acte politique remarquable qui semblait contrer toute forme de nationalisme exacerbé, se distanciant des politiques fascistes, bien avant que la diversité linguistique ne devienne le cheval de bataille de l'Union européenne.

Toutefois, les dispositions constitutionnelles du Statut ont été appliquées avec beaucoup de retard à l'école maternelle et dans les écoles primaires, et seulement au cours des dernières années au secondaire. Dans l'école bilingue valdôtaine, en dépit de la parité officiellement déclarée, c'était l'italien qui semblait effectivement prédominer, à cause de l'attribution au français d'une fonction anti-italienne, comme le remarque Balboni (1983, p. 143-144). En fait, malgré le Statut, la langue française n'avait pas repris le rôle d'autrefois dans la vie quotidienne de la collectivité valdôtaine. La situation linguistique de la Vallée d'Aoste d'alors est ainsi décrite par Janin (1991, p. 577) : « l'on apprend le français à l'école, mais l'on parle le patois à la maison, et l'italien dans la vie publique ». Plus tard, l'Atlas de la langue française précise : "En 1994, l'italien domine dans les agglomérations urbaines où l'immigration s'est concentrée, l'usage du franco-provençal existe dans les communes rurales et le français demeure l'apanage d'une certaine aristocratie culturelle et de l'intelligentsia autochtone " (Rossillon, 1995, p. 64). Du moins, le bilinguisme était un trilinguisme avec le francoprovençal.

Pendant ce temps, le mouvement " arpitan », né dans les années 1970, incitait le peuple valdôtain à la guerre pour la libération nationale, prenant le patois comme instrument et étendard de cette lutte. Dans ce mouvement socioculturel, le divorce entre le patois et le français, considéré désormais comme langue colonisatrice aussi bien que l'italien, est énoncé pour la première fois (Omezzoli, 1995). Jusqu'alors, le rapport français/francoprovençal était un rapport de sujétion du francoprovençal par rapport au français : la conviction que la langue française était la langue maternelle des valdôtains n'avait jamais été remise en cause. La situation avait graduellement évolué : le français ne pouvait plus être considéré comme la langue maternelle des Valdôtains mais, au contraire, c'était le francoprovençal qui jouissait de ce statut, le français devenant «langue naturelle » (Bétemps, 1974, p. 8-9). Depuis lors, comme le remarque Berruto (1983, p. 86), l'identité francophone, très forte dans la communauté valdôtaine, pousse vers le français ou plutôt vers le francoprovençal (si le français est perçu comme langue colonisatrice), ou mieux vers les deux langues : le patois, langue maternelle, et le français, langue naturelle.

En même temps, pendant l'après-guerre, on observe de plus en plus une ouverture d'esprit européen, une attitude post-nationaliste, qui calme un peu la guerre des langues. En Europe, à partir de la deuxième moitié des années 1970, dépassé le préjugé monolingue gommant la diversité, qu'elle soit de caractère linguistique, ethnique, régional, religieux, on a compris qu'une langue est un patrimoine culturel et un facteur d'identité communautaire très important qu'il faut sauvegarder et valoriser (Toso, 2008). C'est surtout à partir des années 1990 que l'on a enregistré une attention envers les petites communautés linguistiques et que l'on a reconnu l'importance de préserver la multiplicité culturelle et linguistique. Une étape très importante dans ce sens est représentée par la Charte européenne des langues régionales et minoritaires, promue par le Conseil de l'Europe en 1992, qui mentionne toutes les garanties que les États doivent offrir pour le développement des droits culturels et linguistiques des différentes communautés minoritaires (Carli, 2004). À partir de cette date, les programmes de l'Union européenne portent une attention particulière aux langues européennes menacées de disparition et à la diversité linguistique au sein des États membres, s'engageant en faveur de la sauvegarde et de la promotion de l'exceptionnelle diversité du patrimoine linguistique et culturel de l'Union, en fonction de la reconnaissance d'une identité collective plurilingue et pluriculturelle (Orioles, 2003; Pizzorusso, 2001).

Dans le cadre de ce mouvement européen, la promotion du patois connaît des conditions favorables. 


\section{La loi 482/1999 et la reconnaissance juridique du francoprovençal}

En Italie, c'est la loi du 15 décembre 1999, $\mathrm{n}^{\circ}$ 482, Règles en matière de protection des minorités linguistiques historiques, qui donna une impulsion ultérieure aux initiatives à l'appui du francoprovençal. À partir de ce moment, l'attention de l'Administration régionale de la Vallée d'Aoste se focalisa notamment sur la promotion du patois, et ce, au détriment du français (Celi, 2015).

La loi 482/1999, qui applique après plus d'un demi-siècle l'article 6 de la Constitution de 1948 : « La République protège par des normes particulières les minorités linguistiques ", énonce, dans son article 2, l'engagement à protéger douze langues minoritaires de vieil enracinement présentes en Italie :

En vertu de l'article 6 de la Constitution et en harmonie avec les principes généraux établis par les organisations européennes et internationales, la République protège la langue et la culture des populations albanaise, catalane, germanique, grecque, slovène et croate, et de celles qui parlent le français, le franco-provençal, le frioulan, le ladin, l'occitan et le sarde.

Reconnaissant le français et le francoprovençal comme langues minoritaires de l'État italien, cette loi nationale semble refléter la volonté politique d'harmoniser les dispositions nationales avec l'esprit des accords internationaux sur lesquels se fonde le bilinguisme en Vallée d’Aoste.

\section{La situation actuelle}

La situation actuelle est plus ou moins la même que celle de l'immédiat après-guerre, quand le français a été réintroduit par le biais du système scolaire. Aujourd'hui, l'usage réel du français est très restreint, limité à des contextes bien précis, et observe un déclin constant depuis la période fasciste. La politique linguistique de la Vallée d'Aoste charge presque exclusivement l'enseignement de préserver le bilinguisme officiel, là où l'italien et le patois sont appris au sein des familles. C'est une donnée importante pour les responsables de la politique linguistique, qui émerge du sondage de la Fondation Chanoux : pour le français, le domaine qui semble le plus découvert est celui de la famille et le plus couvert, celui de l'école. Dans le cas du francoprovençal, les rapports s'inversent, car c'est la famille qui semble être la plus active. Cette donnée explique le maintien de cette langue en Vallée d'Aoste à la différence du français, qui fait pourtant l'objet d'une politique linguistique (Cavalli, 2003). L'Institut Régional de Recherche Éducative relève à propos de la politique linguistique du français: "La langue reste valorisée linguistiquement mais n'est pas valorisée dans le domaine de culture générale, vie quotidienne, compréhension de disciplines nonlinguistiques " (Coste, Sobrero, Cavalli et Bosonin, 2006, p. 69). À propos de la Vallée d'Aoste, on parle en effet de bilinguisme éducatif, « du fait que l'école est le contexte de référence pour l'apprentissage et l'acquisition du français" (Decime et Vernetto, 2007, p. 46). Le français, solidement enraciné dans les écoles valdôtaines comme matière linguistique, souffre du manque de véhicularité et d'utilisation dans les autres domaines. Le francoprovençal, par contre, soutenu aujourd'hui par le gouvernement de la région ainsi que par quelques associations culturelles telles que le Comité des Traditions Valdôtaines, la Fondation Émile Chanoux et la Société des Recherches et d'Études francoprovençales, est une langue encore bien vivante, surtout dans les vallées de la moyenne montagne. Reprenant la synthèse proposée par Josserand (2011, p. 92), le francoprovençal est utilisé en famille, au travail et dans le champ des traditions populaires, le français, à l'église, dans l'enseignement de la langue française ou de la culture valdôtaine, et l'italien est utilisé dans tous les domaines.

Selon Bétemps (1998, p. 159), "le français est encore la langue maternelle d'un petit nombre de familles valdôtaines ", là où le patois est celle d'un nombre considérable de Valdôtains. Plus précisément, le sondage linguistique de la Fondation Chanoux relève que 71,6 \% des Valdôtains sont italophones - qui plus est, presque la totalité des plus jeunes se disent de langue maternelle italienne - et $16 \%$ environ de la population a le patois comme langue maternelle (Tessarolo, 2003, p. 122). Une autre donnée alarmante : la plupart des Valdôtains (79\%) estiment que l'italien est la langue qui remplit toutes les fonctions vitales dans la communauté, alors que le français et le francoprovençal n'ont pas d'utilité pratique dans leur vie quotidienne, même s'ils croient qu'il est important de connaître ces deux langues (Cavalli, 2003, p. 20). Selon une enquête conduite par Josserand (2003), les personnes de plus de 55 ans se considèrent comme Valdôtains avant tout et ils montrent un sentiment identitaire assez froid par rapport à l’État italien alors que les jeunes mettent davantage en avant leur identité italienne. De 
plus, les personnes de plus de 55 ans utilisent dans la vie de tous les jours le francoprovençal, ce qui révèle une assise identitaire forte, qui n'existe pas chez les jeunes. Pour les plus âgés, le français n'a pas d'importance sociale mais il est considéré comme dépositaire d'une base historique importante. Chez les jeunes, en revanche, ni l'un ni l'autre ne sont considérés comme nécessaires, même on reconnaît leur importance culturelle et historique. D'après l'enquête de Puolato (2006, p. 356), l'utilité du français s'avère limitée à la proximité physique avec la France et la Suisse romande. Il semble que la plupart des interrogés aient de moins en moins tendance à utiliser le français comme langue véhiculaire : ils préfèrent apprendre une autre langue : l'anglais avant tout.

On est passé, donc, d'une situation (jusqu'à l'Unité italienne) où la langue française était la langue-toit à une situation où la prédominance de la langue-toit nationale, qui a totalement remplacé le français dans tous les contextes, est devenue irréversible. À la lumière des données relevées jusqu'ici, le bilinguisme officiel est en fait représenté par le binôme italien/francoprovençal : dans les faits, la plupart des Valdôtains sont bilingues italienfrancoprovençal, même si tous connaissent le français au moins à un niveau moyen. Pour une partie considérable des Valdôtains, le français est langue seconde, ses emplois sont extrêmement restreints et il occupe une place surtout symbolique, liée à son passé : très peu de Valdôtains l'utilisent et reconnaissent la fonction identitaire du patois (Berruto, 2003, p. 44). En effet, le francoprovençal vit une récupération culturelle et resurgit comme langue identitaire et symbolique, se conjuguant tantôt avec la défense du français, tantôt en opposition au français (Cavalli, 1997).

L'intérêt réel pour la survie de la diversité linguistique semble très faible et on perçoit plutôt l'avantage assuré par le bilinguisme officiel, à savoir la possibilité de se définir "autres " et, par conséquent, de maintenir un Statut d'autonomie très utile du point de vue financier (Iannaccaro, Dell'Aquila et Andrione, 2003, p. 9-10). On a l'impression que la langue française n'est maintenue que pour des raisons politiques, étant donné que l'autonomie accordée à la Vallée d’Aoste dans le cadre du Statut Spécial d'autonomie repose uniquement sur cette particularité linguistique.

\section{Conclusions et perspectives}

Force est donc de constater que le francoprovençal et le français courent le risque concret de disparaître en Vallée d'Aoste puisque si, d'un côté, le premier jouit encore d'une importance sociale, de l'autre côté, il ne possède pas vraiment de patrimoine littéraire ; le français, par contre, possède un patrimoine culturel important, mais il n'est pas perçu comme tel au niveau local valdôtain, et ne possède aucune importance sociale. La progression constante de l'italien, langue première de la majorité des Valdôtains aux dépens du français et du francoprovençal, est acceptée par les jeunes et elle est désormais vue comme quelque chose de naturel. C'est le résultat de la gestion du "plurilinguisme de fait» (Gohard-Radenkovic, 2010, p. 125) par les institutions valdôtaines, des choix de politique linguistique, que Calvet définit « in vitro » pour les opposer à la gestion " in vivo » : "Si les choix in vitro prennent le contre-pied de la gestion in vivo (gestion par le peuple) ou des sentiments linguistiques du locuteur, il sera difficile d'imposer à un peuple une langue nationale dont il ne veut pas » (Calvet, 1993, p. 112).

Comme nous l'avons vu, le bilinguisme de type éducatif n'est pas suffisant pour assurer la survie d'une langue. Dans le domaine de l'école, il s'avère nécessaire de promouvoir une politique linguistique reposant sur un concept d'enseignement qui permette à tous les citoyens d'utiliser leur langue dans le plus grand nombre de contextes possibles, encourageant ainsi un plurilinguisme qui exclut que l'apprentissage d'une langue puisse se faire avec I'intention d'en discriminer une autre (Nelde, 2003, p. 73). Les politiques linguistiques doivent encourager la transmission intergénérationnelle, qui est, selon les sociolinguistes, la seule mesure efficace pour faire revivre une langue. Dans ce sens, la politique linguistique doit favoriser le français dans des domaines autres que l'école, tels que la famille, les médias et l'administration. Selon Cavalli (1997, p. 90), il faut aussi « concilier, d'un côté, la sauvegarde des droits des Valdôtains revendiquant le français et, de l'autre, la nécessité de créer le consensus auprès de ceux que s'y opposent ». Quant au francoprovençal, il faut notamment éliminer les obstacles qui en réduisent les domaines d'utilisation, essayant d'améliorer les attitudes, les représentations de cette langue, à travers une revalorisation de la culture montagnarde et paysanne, la reconnaissance de son importance comme langue du territoire, langue patrimoniale dont la population peut être fière. Selon Strubell (2003, p. 112), « c'est la vitalité du francoprovençal qui pourra sauver le français dans la Vallée : pas à l'inverse ! ». 
Dans le but de sauvegarder le particularisme valdôtain et, en même temps, de respecter les exigences réelles de la communauté valdôtaine, la politique doit fournir les moyens pour réaliser des actions visant la protection et la valorisation du patrimoine et le respect des droits de l'Homme, qui puissent retomber positivement sur la société tout entière. Dans cette optique, tous doivent contribuer à l'épanouissement de la personnalité de la Vallée d'Aoste et à la conservation de son originalité, de son particularisme.

D'ailleurs, le discours sur la diversité linguistique et culturelle a radicalement changé : la diversité ne représente plus une menace pour les identités nationales, religieuses et culturelles, mais elle est considérée comme une richesse, un bien commun de l'humanité, une valeur universelle. En particulier, la Déclaration universelle de I'Unesco sur la diversité culturelle, adoptée en 2001, affirme que la défense de la diversité culturelle doit être « un impératif éthique, inséparable du respect de la dignité de la personne humaine ». La prise en compte et le développement du potentiel plurilingue de chaque individu est l'un des principes que le Conseil de l'Europe prône avec force.

Selon l'article 1 de la loi régionale 18/2005 : « Le caractère bilingue de l'école valdôtaine représente la garantie du développement d'une éducation plurilingue, ouverte à l'Europe et visant également au respect des différences culturelles et linguistiques » (Conseil de la Vallée d'Aoste).

En 2009, suivant les orientations en matière de plurilinguisme que le Conseil de l'Europe (2007) a présentées dans le Guide pour l'élaboration des politiques linguistiques éducatives, la Vallée d'Aoste a établi son Profil de la politique linguistique éducative, qui propose des pistes de solution et des suggestions. Aujourd'hui, la politique linguistique éducative de la région, qui va dans le sens d'une éducation plurilingue et vers un affermissement du plurilinguisme, vise, d'un côté, à la promotion de la diversité linguistique et culturelle qui la caractérise et, de l'autre, à l'amélioration et à la consolidation des compétences linguistiques des élèves (Vernetto, 2016). Se basant sur le choix politique de considérer le plurilinguisme comme une valeur au service de la citoyenneté européenne, le Profil a mis en œuvre toute une série d'actions pour promouvoir le multilinguisme de la société valdôtaine ainsi que le potentiel plurilingue de chaque individu. Dans ce cadre, le système valdôtain accorde au français un « rôle pivot dans la formation et l'éducation plurilingues" (Decime et Vernetto, 2009, p. 59). Certes, il faut attendre encore pour voir les résultats concrets de la mise en place de ce projet. Par ailleurs, comme le souligne Gohard-Radenkovic (2012, p. 90), l'association de la diversité linguistique avec le plurilinguisme est désormais une idéologie affichée noir sur blanc par le Conseil de l'Europe dans le titre de son Guide pour l'élaboration des politiques linguistiques éducatives en Europe: "De la diversité linguistique à l'éducation plurilingue ». En partageant les conclusions de Calvet (2002, p. 46), selon qui « ce respect de la diversité est un jeu à somme nulle dans lequel les gains et les pertes s'annulent ", Gohard-Radenkovic (2012) souligne les dangers éventuels de la promotion de la diversité, dans la mesure où la promotion d'une langue se fait souvent au détriment des autres. En effet, si le plurilinguisme et le respect de la diversité linguistique et culturelle sont imposés, ils produisent souvent des résultats tout à fait opposés aux finalités.

C'est le risque que court la Vallée d'Aoste où un plurilinguisme institutionnalisé pourrait ne pas répondre aux enjeux des jeunes générations.

Le moyen le plus sûr d'éviter ce danger réel, c'est de transmettre les langues à nos enfants. Nous avons le devoir de ne pas les priver de cette richesse extraordinaire! Voilà pourquoi il faut favoriser l'ouverture aux langues et aux cultures, non seulement à celles que l'école a la vocation d'enseigner, mais aussi à celles de l'environnement familial et social des enfants. Cela est d'autant plus indispensable dans le contexte social multilingue valdôtain, où l'école est obligée de prendre en compte la diversité des langues et des cultures présentes dans l'environnement de la classe. II s'agit donc de promouvoir une éducation au plurilinguisme par des méthodologies et des supports qui permettent de s'appuyer sur les compétences linguistiques des élèves pour les amener à découvrir le fonctionnement des autres langues et à développer des stratégies d'apprentissage, permettant ainsi à l'enfant de construire son identité en contact avec l'altérité. C'est par la pratique des "approches plurielles " (Candelier, 2007), à partir de l'école de l'enfance, que l'on peut atteindre ces objectifs: l'éveil aux langues, l'intercompréhension entre langues apparentées, l'approche interculturelle et la didactique intégrée des langues 
valorisent l'idiome d'origine des apprenants et ouvrent leur horizon à d'autres langues et cultures, suscitant des attitudes positives envers d'autres systèmes linguistiques.

En particulier, le dispositif Sacs d'histoires ${ }^{4}$, intégrant toutes ces approches, favorise la prise en compte et la valorisation des langues de l'environnement de l'enfant par l'implication des parents dans la transmission des langues familiales. II permet ainsi l'apprentissage précoce des langues et le lien entre l'école et la famille. Exploitant l'approche de l'intercompréhension ${ }^{5}$, où chacun s'engage à comprendre la langue de l'autre et à se faire comprendre dans sa ou ses langue(s) romane(s) de référence, on combat l'uniformisation et la perte de la diversité. L'enseignement du francoprovençal s'inscrit justement dans une approche de didactique intégrée des langues et se fait en liaison avec l'enseignement du français, en raison de l'appartenance de ces deux langues au domaine galloroman. De plus, le français qui, parmi les langues romanes, a la plus grande proximité géographique, lexicale et syntaxique avec l'anglais, favorise aussi l'apprentissage de cette langue.

C'est ainsi que tous les Valdôtains, reconnaissant les chances que leur offre le plurilinguisme dans un nouveau discours européen qui ne les oblige plus, comme autrefois, à renier leur identité et à se fondre dans les langues de prestige, peuvent se sentir directement concernés par la protection et la promotion de la diversité linguistique. Dans le cadre d'un plurilinguisme, dont la vision et la conception soient réellement partagées par tous, le français et le francoprovençal en Vallée d'Aoste pourront retrouver sans doute leur rôle social. C'est dans cette perspective que, grâce à l'accès à des financements européens, le projet " Lectŭrĭo + ", dont nous sommes partenaires, pourra apporter une contribution significative.

\section{Références bibliographiques}

Ascoli, G.I. (1878). Schizzi franco-provenzali. Archivio Glottologico Italiano, 3, 61-120.

Balboni, P. (1983). La Val D’Aosta. Dans G. Freddi (dir.), L'Italia plurilingue (p. 126-151). Bergamo : Minerva Italica.

Bauer, R. (1999). Storia della copertura linguistica della Valle d'Aosta dal 1860 al 2000 : un approccio sociolinguistico. Nouvelles du Centre d'Études Francoprovençales « René Willien », 39, 76-96.

Berruto, G. (1983). Aspetti e problemi del plurilinguismo in Valle d'Aosta. Dans F. Di lorio (dir.), L'educazione plurilingue in Italia (p. 77-101), Frascati : Quaderni di Villa Falconieri.

Berruto, G. (2003). Una Valle d'Aosta, tante Valli d'Aosta ? Considerazioni sulle dimensioni del plurilinguismo in una comunità regionale. Dans G. Iannaccaro, V. Dell'Aquila et E. Andrione (dir.), Une Vallée d'Aoste bilingue dans une Europe plurilingue / Una Valle d'Aosta bilingue in un'Europa plurilingue (p. 44-53). Aoste : Fondation Émile Chanoux.

Bétemps, A. (1974). Franco-provençal et français en Vallée d'Aoste : une analyse des perturbations intervenues dans leurs relations. Le Flambeau, 1, 5-17.

Bétemps, A. (1978). Réflexions sur le patois et le français en Vallée d’Aoste. Le Flambeau, 2, 5-17.

Bétemps, A. (1979). Les Valdôtains et leur langue. Aoste : Union Valdôtaine.

Bétemps, A. (1998). La situation linguistique valdôtaine. Dans E. Cason Angelini (dir.), "Mes Alpes à moi ». Civiltà storiche e comunità culturali delle Alpi (p. 159-164). Belluno : Regione del Veneto.

Calvet, J.-L. (1987). La guerre des langues et les politiques linguistiques. Paris : Payot.

Calvet, J.-L. (1993). La sociolinguistique. Paris : Presses universitaires de France.

Calvet, J.-L. (2002). Le marché aux langues. Paris : Plon.

\footnotetext{
${ }^{4}$ Née en 1997 en Angleterre, c'est en Suisse (2007) que l'initiative « sac d'histoires » est devenue porteuse de plurilinguisme. Depuis lors, le concept s'est répandu au Luxembourg, en France et en Vallée d’Aoste, où le projet Sacs d'histoires, « Des contes dans nos langues ", a été lancé en 2011. Voir à ce propos Vernetto (2017).

5 Pour un approfondissement sur ce sujet, voir De Carlo (2011).
} 
Candelier, M. (dir.). (2007). CARAP - Cadre de Référence pour les Approches Plurielles des Langues et des Cultures. Graz : Centre européen pour les langues vivantes / Conseil de l’Europe.

Carli, A. (2004). Plurilinguismo e lingue minoritarie nella politica linguistica europea. Revue française de linguistique appliquée, 9, 59-79.

Cavalli, M. (1997). Représentations sociales et politique linguistique. Le cas du Val d’Aoste. Tranel, $27,83-97$.

Cavalli, M. (2003). Bilinguisme et plurilinguisme au Val d'Aoste : le rôle de l'école. Premières réflexions à partir des résultats du sondage linguistique. Dans G. Iannaccaro, V. Dell'Aquila et E. Andrione (dir.), Une Vallée d'Aoste bilingue dans une Europe plurilingue / Una Valle d'Aosta bilingue in un'Europa plurilingue (p. 18-25). Aoste : Fondation Émile Chanoux.

Celi, A. (2015). La « langue du cœur ». Metamorfosi e usi politici di francese e francoprovenzale in Valle d'Aosta (1930-2012). Dans M. Di Giacomo, N. Di Nunzio, A. Gori et F. Zantedeschi (dir.), Piccole tessere di un grande mosaico. Nuove prospettive dei regional studies (p. 341-358). Roma : Aracne.

Conseil de la Vallée d’Aoste (2005). Loi régionale $n^{\circ} 18$ du 1er août 2005. http://www.consiglio.regione.vda.it/fr/app/leggieregolamenti/dettaglio?pk_Ir=3141

Conseil de l'Europe (2007). Guide pour l'élaboration des politiques linguistiques éducatives en Europe. http://www.coe.int/T/DG4/linguistic/Guide_niveau3_FR.asp

Coste, D., Sobrero, A., Cavalli, M. et Bosonin I. (2006). Multilinguisme, plurilinguisme, éducation. Les politiques linguistiques éducatives. Aoste : Institut Régional de Recherche Éducative de la Vallée d'Aoste.

De Carlo, M. (dir.). (2011). Intercomprensione e educazione al plurilinguismo, Fermo : Wizarts.

Decime, R. et Vernetto, G. (2007). Profil de la politique linguistique éducative. Vallée d'Aoste : Rapport Régional. Aoste : Assessorat à l’Éducation et à la Culture.

Decime, R. et Vernetto, G. (2009). Profil de la politique linguistique de la Vallée d'Aoste. Aoste : Le Château.

Fondation Émile Chanoux. http://www.fondchanoux.org/recherchelinguistique.aspx, consulté le 10 mai 2017.

Gohard-Radenkovic, A. (2010). Politiques de gestion de la pluralité linguistique : leurs effets sur les logiques des institutions et les logiques des individus. Dans F. Ruegg et A. Boscoboinik (dir.), From Palermo to Penang. A Journey into Political Anthropology / De Palerme à Penang. Un itinéraire en anthropologie politique. Hommage à Christian Giordano (p. 119-135). Berlin : LIT Verlag.

Gohard-Radenkovic, A. (2012). Le plurilinguisme, un nouveau champ ou une nouvelle idéologie ? Ou quand les discours politiquement corrects prônent la diversité. Alterstice, 2(1), 89-102.

Iannaccaro, G., Dell'Aquila, V. et Andrione, E. (dir.) (2003). Une Vallée d'Aoste bilingue dans une Europe plurilingue / Una Valle d'Aosta bilingue in un'Europa plurilingue. Aoste : Fondation Émile Chanoux.

Janin, B. (1991). Une région alpine originale. Le Val d'Aoste. Tradition et Renouveau. Aoste : Mesumeci.

Josserand, J.-F. (2003). Conquête, survie et disparition : italien, français et francoprovençal en Vallée d'Aoste. Uppsala : Acta universitatis Upsaliensis.

Josserand, J.-F. (2011). Langue et identité : abandon ou maintien d'une langue, le cas de la Vallée d'Aoste. Synergies. Pays scandinaves, 6, 89-96.

Nelde, P. H. (2003). Les minorités et le nouveau plurilinguisme. Dans G. Iannaccaro, V. Dell'Aquila et E. Andrione (dir.), Une Vallée d'Aoste bilingue dans une Europe plurilingue / Una Valle d'Aosta bilingue in un'Europa plurilingue (p. 68-75). Aoste : Fondation Émile Chanoux.

Omezzoli, T. (1995). Lingue e identità valdostana. Dans S. Woolf (dir.), Storia d'Italia : le regioni dall'Unità ad oggi. La Valle d'Aosta (p. 137-202). Torino : Einaudi.

Orioles, V. (2003). Le minoranze linguistiche. Profili sociolinguistici e quadro dei documenti di tutela. Rome : II Calamo.

Alterstice - Revue Internationale de la Recherche Interculturelle, vol. $7, n^{\circ} 2$ 
Pizzorusso, A. (2001). Legislazioni europee sulle lingue minoritarie. Lingua e stile, 36(2), 211-217.

Puolato, D. (2006). Francese-italiano, italiano-patois : il bilinguismo in Valle d'Aosta fra realtà e ideologia. Berne : Peter Lang.

République italienne (1999). Loi du 15 décembre 1999, $n^{\circ}$ 482. Règles en matière de protection des minorités linguistiques historiques. http://portal-lem.com/images/fr/Italie/Loi_482_du_15_decembre_1999.pdf

Rossillon, P. (dir.). (1995). Atlas de la langue française. Paris : Bordas.

Sénat de la République italienne. Constitution de la République italienne [1947]. https://www.senato.it/documenti/repository/istituzione/costituzione_francese.pdf

Schüle, E. (1990). Histoire linguistique de la Vallée d'Aoste. Nouvelles du Centre d’Études Francoprovençales « René Willien », 22, 5-19.

Strubell, M. (2003). Implications du sondage pour la planification linguistique dans la Vallée d'Aoste. Dans G. Iannaccaro, V. Dell'Aquila et E. Andrione (dir.), Une Vallée d'Aoste bilingue dans une Europe plurilingue / Una Valle d'Aosta bilingue in un'Europa plurilingue (p. 105-112). Aoste : Fondation Émile Chanoux.

Tessarolo, M. (2003). Vantaggi delle regioni plurilingui. Il caso della Valle d'Aosta. Dans G. Iannaccaro, V. Dell'Aquila et E. Andrione (dir.), Une Vallée d'Aoste bilingue dans une Europe plurilingue / Una Valle d'Aosta bilingue in un'Europa plurilingue (p. 121-125). Aoste : Fondation Émile Chanoux.

Toso, F. (2008). Le minoranze linguistiche in Italia. Bologna : Il Mulino.

Tuaillon, G. (1992). Histoire des situations linguistiques le long de la frontière franco-italienne. Nouvelles du Centre d’Études Francoprovençales « René Willien », 25, 5-19.

Unesco (2001). Déclaration universelle de l'Unesco sur la diversité culturelle. http://portal.unesco.org/fr/ev.phpURL_ID=13179\&URL_DO=DO_TOPIC\&URL_SECTION=201.html

Vernetto, G. (2016). Le Profil de la politique linguistique éducative de la Vallée d’Aoste : retombées et perspectives. Repères DoRiF, 11.

Vernetto, G. (2017). Les sacs d'histoires : mode d'emploi. Éducation et sociétés plurilingues / Educazione e società plurilingui, 43, 19-31.

Zanotto, A. (1986). Le particularisme valdôtain. Aperçu historique. Aoste : Région Autonome de la Vallée d'Aoste. 